\title{
Resveratrol attenuates neurological deficit and neuroinflammation following intracerebral hemorrhage
}

\author{
JIA-CHEN CAI ${ }^{1}$, WEI LIU ${ }^{2}$, FEI LU ${ }^{3}$, WEN-BING KONG ${ }^{4}$, XIN-XUAN ZHOU ${ }^{4}$, \\ PENG MIAO ${ }^{4}$, CHENG-XIANG LEI ${ }^{5}$ and YAN WANG ${ }^{6}$
}

\author{
${ }^{1}$ Department of Neurosurgery, The No. 2 People's Hospital of Changshu, Suzhou, Jiangsu 215500; \\ ${ }^{2}$ Department of Neurosurgery, The Affiliated Hospital of Qingdao University, School of Medicine, Qingdao University, \\ Qingdao, Shandong 266000; ${ }^{3}$ Key Laboratory of Chemical Genomics, School of Chemical Biology and Biotechnology, \\ Peking University Shenzhen Graduate School, Shenzhen, Guangdong 518055; ${ }^{4}$ Department of Neurosurgery, \\ East District of Qingdao Municipal Hospital, School of Medicine, Qingdao University, Qingdao, \\ Shandong 266000; ${ }^{5}$ Department of Biomedical Sciences, Institute of Molecular Medicine, HuaQiao University, \\ Quanzhou, Fujian 362021; ${ }^{6}$ Department of Cardiology, East District of Qingdao Municipal Hospital, \\ School of Medicine, Qingdao University, Qingdao, Shandong 266000, P.R. China
}

Received November 1, 2016; Accepted October 6, 2017

DOI: $10.3892 / \mathrm{etm} .2018 .5938$

\begin{abstract}
Resveratrol (RESV) improves histopathological and behavioral outcomes in diseases of the central nervous system. However, to the best of our knowledge, there have been no studies investigating its neuroprotective effects on secondary brain injury following intracerebral hemorrhage (ICH). The aim of the present study was to evaluate the neuroprotective function of resveratrol following ICH. Male Sprague-Dawley rats were randomly divided into 3 groups: Sham, ICH and $\mathrm{ICH}+\mathrm{RESV}$ groups. Rats underwent $\mathrm{ICH}$ and received an intraperitoneal injection of RESV daily. Rotarod and open field tests were performed to evaluate improvements in motor disturbance pre- and post-surgery. Rats were sacrificed following the final behavioral test; subsequently, neuron injury and cell death in the hippocampus and microglia activation in the cortex were determined using Nissl staining and ionized calcium binding adaptor molecule 1 immunofluorescence
\end{abstract}

Correspondence to: Dr Wei Liu, Department of Neurosurgery, The Affiliated Hospital of Qingdao University, School of Medicine, Qingdao University, 6 Jiangsu Road, Qingdao, Shandong 266000, P.R. China

E-mail: surg.liuwei@126.com

Dr Fei Lu, Key Laboratory of Chemical Genomics, School of Chemical Biology and Biotechnology, Peking University Shenzhen Graduate School, Peking University Campus F(East)302/309, Shenzhen University Town, Lishui Road, Xili Town, Shenzhen, Guangdong 518055, P.R. China

E-mail: lufei@pkusz.edu.cn

Key words: resveratrol, intracerebral hemorrhage, secondary brain injury, neuroprotection, neuroinflammatory response, behavior test staining, respectively. Compared with the $\mathrm{ICH}$ group, rats treated with resveratrol for 2 weeks performed significantly better in behavioral tests. Furthermore, less neural damage in the hippocampus and decreased activation of microglia was observed in the ICH+RESV group. The results of the present study therefore indicate that resveratrol may alleviate secondary brain injury following $\mathrm{ICH}$.

\section{Introduction}

Spontaneous intracerebral hemorrhage (ICH) is a particularly devastating cerebral vascular disease with a high mortality rate. Complications, including enlarged hematoma volumes, edema exacerbation and secondary brain injury, often develop following ICH. Craniotomy to clear blood clots, antioxidants, antithrombin, neutrophil infiltration inhibitors and heme oxygenase inhibitors are used to treat ICH (1-5). However, there are still few effective methods able to prevent secondary brain injury following ICH.

Resveratrol (RESV) is a natural, non-flavonoid, polyphenol compound found in grapes and other berries that can induce pleiotropic effects in vertebrates (6-9). It has been demonstrated that resveratrol exhibits a neuroprotective function, as it ameliorates kainate-induced excitotoxicity (10). Previous studies have revealed that RESV improves neurological functions in various diseases of the central nervous system, including cerebral ischemia/reperfusion (11), acute and secondary spinal injury (12), neurodegenerative diseases (13) and depression (14). It has been suggested that the protective effects of RESV may be mediated through the sirtuin 1, adenosine 5'-phosphate-activated kinase and nuclear factor erythroid 2-related factor pathways (15-17).

The autologous blood perfusion model is used in physiological, pathomorphological and therapeutic research into $\mathrm{ICH}$, as it mimics hypertensive cerebral hemorrhage (18). Therefore the present study investigated the neuroprotective function of RESV using autologous blood perfusion in a rat model of $\mathrm{ICH}$. 
Rat motor disturbance and neural damage/inflammation were assessed using behavioral tests and immunohistochemistry, respectively.

\section{Materials and methods}

Animals. A total of 30 male Sprague-Dawley rats, weighing 300-350 g and aged 55 days, were purchased from the Guangdong Medical Laboratory Animal Center (Guangzhou, China). Rats were cared for in accordance with the Guideline for the Care and Use of Laboratory Animals published by the National Institutes of Health (NIH Publications no. 8023, revised 1978) (19). Rats were housed conventionally (room temperature, $20 \pm 2^{\circ} \mathrm{C}$; humidity, $55 \%$; 15 air changes per $\mathrm{h}$ and a 12-h light-dark cycle) in polycarbonate cages on hardwood bedding and acclimatized for at least 7 days prior to study initiation. Rats were provided with tap water and rodent chow ad libitum. All experimental procedures were approved by the Animal Care and Use Committee of Peking University Shenzhen Graduate School (Shenzhen, China).

Establishing a hypertensive cerebral hemorrhage rat model. Rats were randomly divided into 3 groups: A sham+dimethyl sulphoxide (DMSO) group, an ICH+DMSO group and an ICH+RESV group (all $n=10$ ). ICH was induced using the autologous blood perfusion model. Rats were anesthetized with $1 \%$ sodium pentobarbital $(60 \mathrm{mg} / \mathrm{kg}$, intraperitoneally; Shanghai Longsheng Chemical Co., Ltd., Shanghai, China) and were immobilized in a stereotactic apparatus frame (RWD Life Science Co., Ltd., Shenzhen, China). A 1-mm bur hole was punctured into rat skulls $(1 \mathrm{~mm}$ anterior and $3 \mathrm{~mm}$ lateral to bregma). Fresh blood (100 $\mu \mathrm{l})$ was drawn from rat caudal arteries using a microsyringe and was injected into the caudate putamen (5.5 mm deep to bregma; Fig. 1A). Autologous blood was infused over 10 min using a microinfusion pump (RWD Life Science Co., Ltd.). The needle was slowly withdrawn 40 min following injection to prevent the backflow of infused blood and to allow for hematoma formation. The burr hole was then sealed with bone wax and the wound was sutured. The needle of an empty syringe was inserted into rats in the sham group. Rat body temperature was maintained at $36 \pm 0.5^{\circ} \mathrm{C}$ using a feedback-controlled heating pad. Following the cessation of anesthesia, rats in the ICH+RESV group were treated with $100 \mathrm{mg} / \mathrm{kg}$ RESV in 5\% DMSO (15 mg/ml), administered intraperitoneally once per day. Sham and $\mathrm{ICH}$ groups were administered with the same volume of 5\% DMSO once per day for 14 days. All rats were sacrificed for histopathological staining following competition of all neurological behavior tests. Brains were subsequently removed from the skull and fixed in 10\% neutral formalin buffer (Sigma-Aldrich; Merck $\mathrm{KGaA}$, Darmstadt, Germany) at room temperature for $24 \mathrm{~h}$. Brain damage following surgery to induce ICH was confirmed using 2-3 mm-thick horizontal sections (Fig. 1B and C).

Rotarod test. Rats underwent a rotarod test on the ZB-200 (Chengdu Techman Software Co., Ltd., Chengdu, China) at a velocity of $5 \mathrm{rpm}$, which was subsequently increased to $60 \mathrm{rpm}$ over $55 \mathrm{sec}$. All rats were trained 20 times with a $20 \mathrm{sec}$ interval. The mean duration of the 20 trials was recorded to assess the daily performance of rats. Rats were trained for 8 days prior to ICH surgery. The performance of rats on the final 3 days was recorded and the mean duration was used as a baseline value. The recovery of motor impairment was examined 3, 7 and 14 days following ICH surgery.

Open field test. Rats were exposed to a circular arena $(100 \times 40 \mathrm{~cm})$ constructed from black plywood and the floor was divided into 25 sections. Rats were individually placed in the center of the apparatus and their behavior was recorded for 10 min using a digital camera situated above their head. Tests were performed 1, 7 and 14 days following surgery. The total distance travelled, average speed, number of total rotations and line crossings were analyzed using ANY Maze software (version 4.84; Stoelting Co., Wood Dale, IL, USA).

Tissue processing. Rats were euthanized 14 days following surgery with an intraperitoneal injection of $120-150 \mathrm{mg} / \mathrm{kg}$ sodium pentobarbital and then were perfused intracardially with $4 \%$ paraformaldehyde in PBS. Brains were removed and fixed in $10 \%$ neutral formalin buffer at room temperature for 24 h. Following dehydration with graded ethanol and xylene, brains were embedded in paraffin wax. A rotary mictrotome (RM2255; Leica Microsystems GmbH, Wetzlar, Germany) was utilized to cut 5- $\mu \mathrm{m}$ tissue sections, which were subsequently stored at $4^{\circ} \mathrm{C}$.

Nissl staining. To quantify brain injuries induced via $\mathrm{ICH}$, Nissl staining was performed following the manufacturer's protocol (Beyotime Institute of Biotechnology, Shanghai, China). Paraffin-embedded sections were dewaxed, rehydrated and stained with Cresyl violet (C0117; Beyotime Institute of Biotechnology). Images were captured using an Olympus fluorescence microscope (CKX41; Olympus Corporation, Tokyo, Japan) at a magnification of x400 or x100 and a cooled charge-coupled camera (QICAM 12-bit), and were processed using the QCapture Pro 6.0 program (both QI imaging, Surrey, Canada). To assess whether the number of living neurons differed significantly between the groups, cells in three independent microscopic fields were examined. The mean ratio of normal neurons was calculated from three independent counts and plotted with error bars representing standard deviation. Neurons with round and pale staining nuclei were regarded as surviving, while shrunken neurons with condensed nuclei were regarded as damaged (20).

Immunostaining. To determine the anti-inflammatory effects of RESV, immunofluorescence with anti-ionized calcium binding adaptor molecule 1 (Iba-1) antibodies was utilized to assess microglial activation in the cortex as previously described (21). Slides were deparaffinized, rehydrated $(100,90,80,70$ and $50 \%$ ethanol, and $\mathrm{ddH}_{2} \mathrm{O}$ ) and immersed in $3 \% \mathrm{H}_{2} \mathrm{O}_{2} /$ methanol for $10 \mathrm{~min}$ at room temperature to inactivate endogenous peroxidase. Antigens were heat-retrieved in sodium citrate buffer (10 mM sodium citrate; $0.05 \%$ Tween-20; $\mathrm{pH} 6.0$ ) at $100^{\circ} \mathrm{C}$ for $8 \mathrm{~min}$. Following blocking in $3 \%$ bovine serum albumin (Sigma-Aldrich; Merck KGaA) for $20 \mathrm{~min}$ at room temperature, tissues were incubated with rabbit anti-Iba-1 antibodies (cat. no. 019-19741; 1:500; Wako Pure Chemical Industries, Ltd., Osaka, Japan) overnight at $4^{\circ} \mathrm{C}$. Sections were washed in PBS and incubated with a fluorescein isothiocyanate-conjugated 
A

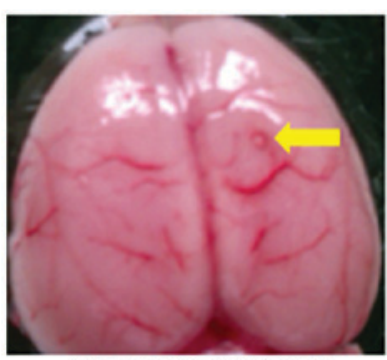

Whole brain tissue
B

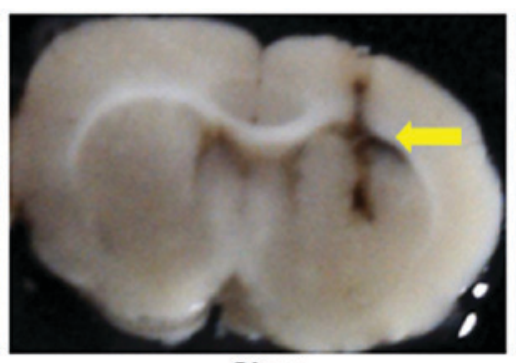

Sham
C

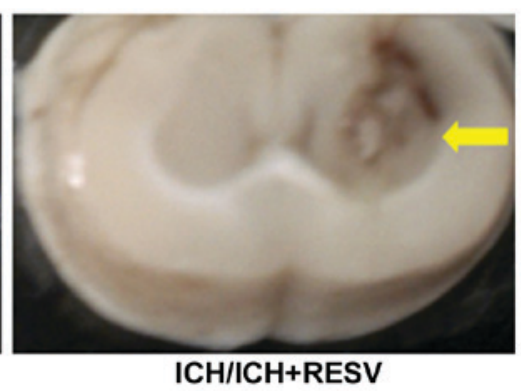

Figure 1. Tissue damage Following ICH surgery. (A) Rat brain tissue presenting the location of the ICH injection. Horizontal brain sections presenting the damage induced by ICH. (B) The brain section of the sham group only exhibits damage at the injection site. (C) The ICH/ICH+RESV group section exhibits a mass of hematoma with serious injury to the basal ganglia. All regions of interested as indicated by yellow arrows. ICH, intracerebral hemorrhage; RESV, resveratrol.
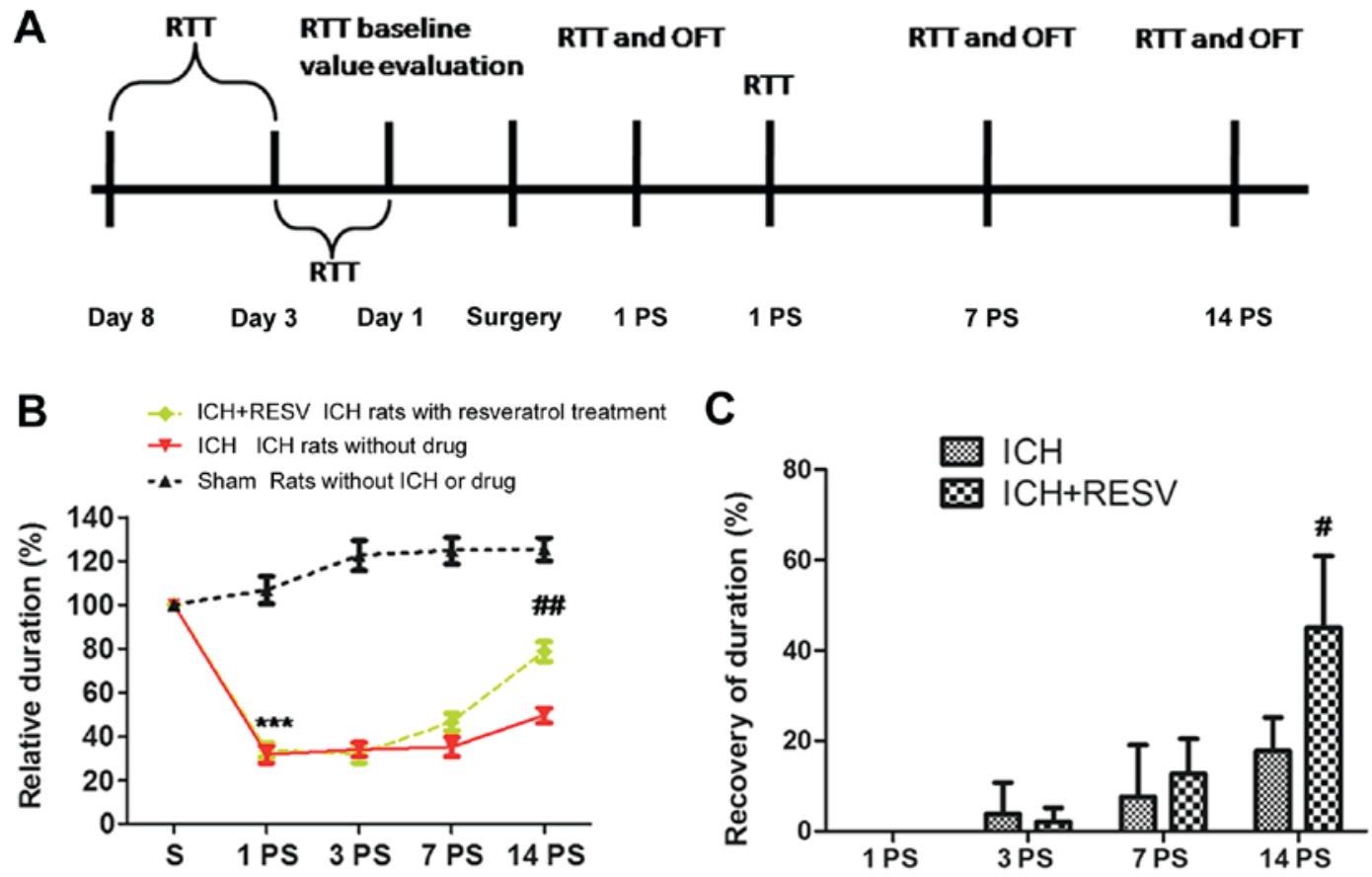

Figure 2. RESV improves the motor abilities of rats in behavioral tests following ICH. (A) Experimental design and behavioral test schedule. (B) RESV improves the motor abilities of rats following $\mathrm{ICH}$, as demonstrated in the rotarod test. The time rats remained on the rotarod from three groups (sham, ICH and ICH+RESV) was recorded as relative duration. (C) Motor recovery was presented as relative duration on the rotarod. ICH rats treated with RESV exhibited a significant recovery compared with untreated ICH rats. Data are presented as the mean \pm standard deviation. ${ }^{* * *} \mathrm{P}<0.001$ vs. sham group, ${ }^{\#} \mathrm{P}<0.05$ and ${ }^{\# \#} \mathrm{P}<0.01$ vs. ICH group. ICH, intracerebral hemorrhage; RESV, resveratrol; RTT, rotarod test; OFT, open field test; S, surgery; PS, days post-surgery.

secondary goat anti-rabbit Immunoglobulin G antibody (cat. no. 111-095-003; 1:50; Jackson ImmunoResearch Europe, Ltd., Newmarket, UK) at room temperature for $1 \mathrm{~h}$. Subsequently, sections were mounted in mowiol mounting medium containing $1 \mu \mathrm{g} / \mathrm{ml}$ DAPI for DNA staining. Images were captured using a fluorescence microscope at a magnification of $\mathrm{x} 400$ or $\mathrm{x} 100$ and a cooled charge-coupled camera, and processed using the QCapture Pro 6.0 software. The images were analyzed using ImageJ 1.42q software (32-bit; National Institutes of Health, Bethesda, MD, USA). To quantify neuroinflammation, cortex Iba-1 positive cells were counted and compared between different groups.

Statistical analysis. The results are presented as the mean \pm standard deviation and all experimental data were analyzed using Prism 5.0 Software (GraphPad Software, Inc.,
La Jolla, CA, USA). Statistical differences among sham, ICH and $\mathrm{ICH}+\mathrm{RESV}$ groups were assessed using one-way analysis of variance followed by the Tukey's test for the comparison of multiple groups. $\mathrm{P}<0.05$ was considered to indicate a statistically significant difference.

\section{Results}

RESV improves motor ability following ICH. To investigate the neuroprotective effects of RESV following $\mathrm{ICH}$, the recovery of rat motor abilities were assessed following ICH (Fig. 2A).

Rotarod tests. The time spent on the rod during the rotarod test was markedly decreased 1 day post-surgery in the ICH and $\mathrm{ICH}+\mathrm{RESV}$ groups, compared with their performance prior to surgery (Fig. 2B). No significant differences in performance 
A

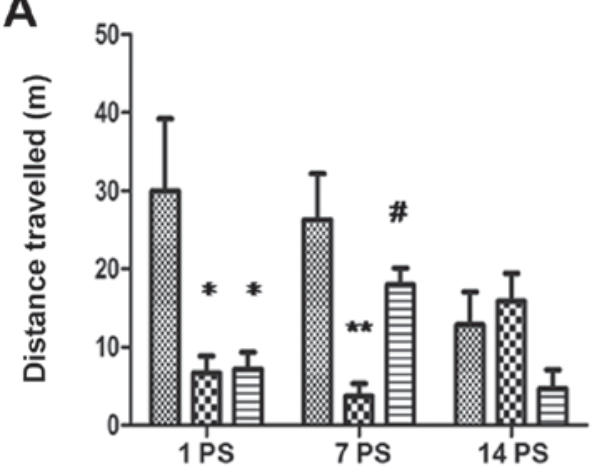

C

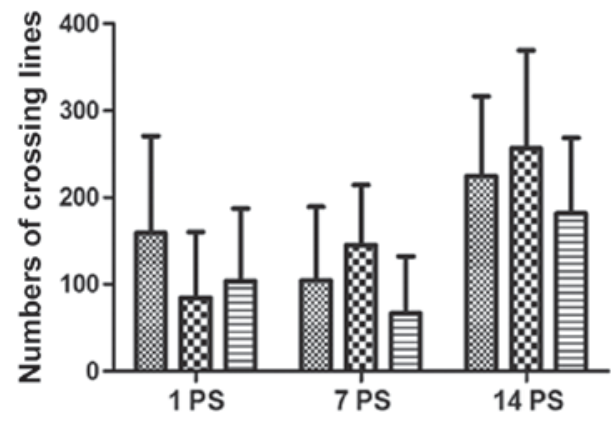

B

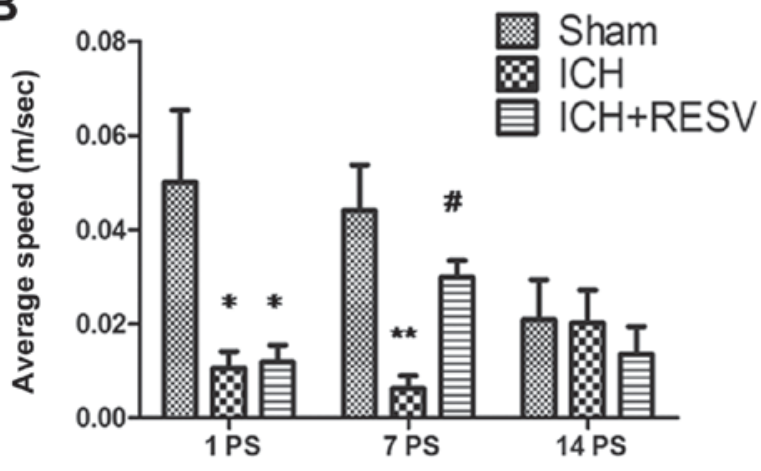

D

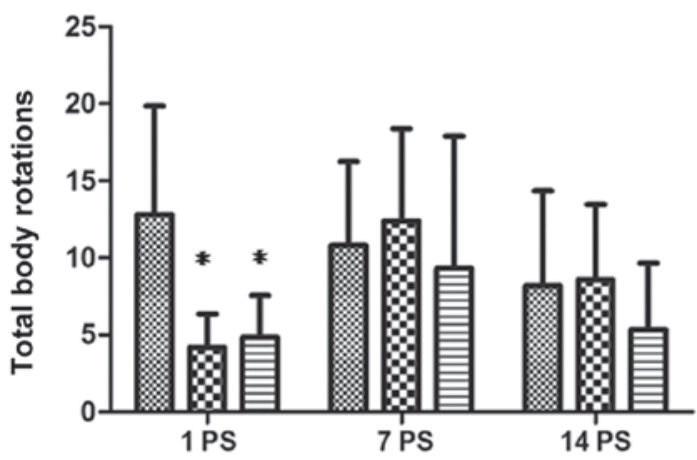

Figure 3. RESV treatment improves rat motor abilities following ICH as demonstrated in open field tests. Four parameters including (A) total distance traveled, (B) average speed, (C) number of lines crossed and (D) total body rotations were evaluated in open field tests 1,7 and 14 days following ICH and RESV administration. Data are presented as the mean \pm standard deviation. ${ }^{*} \mathrm{P}<0.05$ and ${ }^{* *} \mathrm{P}<0.01$ vs. sham group, ${ }^{*} \mathrm{P}<0.05$ vs. ICH group. RESV, resveratrol; ICH, intracerebral hemorrhage; PS, days post-surgery.

were identified between the ICH and ICH+RESV groups on day 1 . A recovery was observed 7 and 14 days post-surgery in the ICH+RESV and ICH groups (Fig. 2B). Sham rats exhibited a small increase in relative duration 1 day post-surgery but subsequently remained steady between 3 and 14 days post-surgery. Although the ICH and ICH+RESV groups exhibited a significant recovery 14 days post-surgery, the $\mathrm{ICH}+\mathrm{RESV}$ group exhibited a significant increase in relative duration compared with the ICH group ( $\mathrm{P}<0.05$; Fig. 2C). The results indicate that RESV accelerates the recovery of rat motor abilities following ICH.

Open field test. Parameters, including the total distance traveled (Fig. 3A), average speed (Fig. 3B), number of lines crossed (Fig. 3C) and total body rotations (Fig. 3D) were evaluated in an open field test 1, 7 and 14 days following ICH and RESV treatment. Compared with the sham group, the distance traveled, average speed and total body rotations were significantly decreased 1 day post-surgery in the ICH and ICH+RESV groups (all $\mathrm{P}<0.05$; Fig. 3A, B and D). Rats in the ICH group travelled shorter distances and moved at slower speeds than the sham group 1-7 days post-surgery $(\mathrm{P}<0.05$; Fig $3 \mathrm{~A}$ and $\mathrm{B})$. No significant differences between the ICH and ICH+RESV groups were identified in any of the tests conducted 1 day post-surgery. In addition, no significant differences in the total number of lines crossed were identified among any of the groups (Fig. 1C). These data indicate that the motor ability of rats is significantly decreased following ICH.

Following RESV administration for 7 days post-surgery, the total distance travelled and average speed of rats in the
ICH+RESV group were significantly increased compared with $\mathrm{ICH}$ rats $(\mathrm{P}<0.05$; Fig. $3 \mathrm{~A}$ and $\mathrm{B})$. However, no significant differences between these groups were identified in the number of lines crossed and total body rotations. These results indicated that the motor abilities of rats had partially recovered following RESV treatment. However, no significant differences were identified in any groups 14 days post-surgery. This may have been due to the habituation of rats to the experimental environment following test repetition. These data demonstrate that RESV treatment may improve the motor abilities of rats following $\mathrm{ICH}$.

RESV alleviates damage to neurons in the hippocampus. Nissl staining was performed to evaluate the morphological changes of neurons in the hippocampus following ICH. Integrated bluish-violet neuronal cells with an articulate structure of mottled nuclei and cytoplasm were observed in the sham group, while condensed and irregular cytons of injured neurons, combined with few normal neurons, were identified in the ICH groups (Fig. 4A). The number of normal neurons significantly decreased following ICH surgery compared with the sham group $(\mathrm{P}<0.001$; Fig. 4B). Following RESV treatment, the number of normal neurons significantly increased $(\mathrm{P}<0.001$; Fig. 4B). These results suggest that RESV treatment alleviates the neuronal damage induced by $\mathrm{ICH}$.

Neuroinflammatory responses are ameliorated by RESV following ICH. Microglia are the resident macrophages of the central nervous system and are the primary form of active immune defense available (22). Following activation 
A

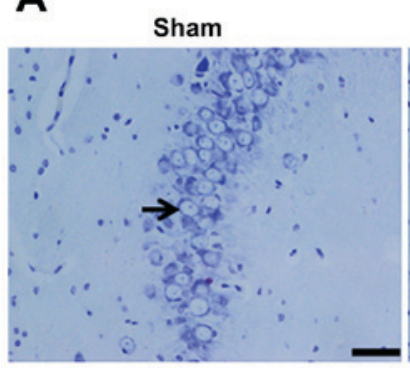

$\mathrm{ICH}$

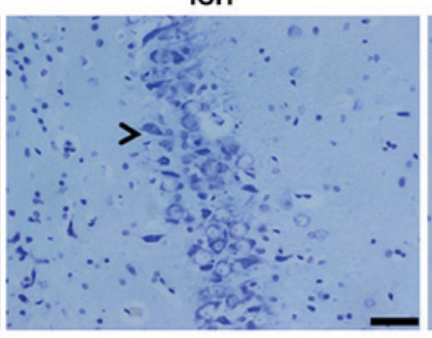

ICH+RESV

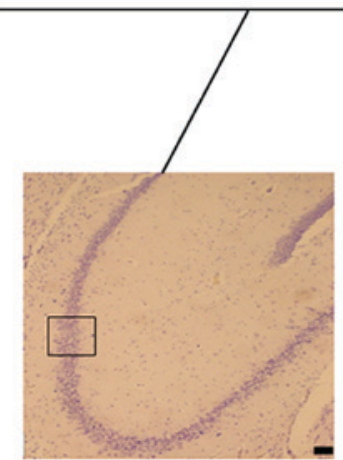

B

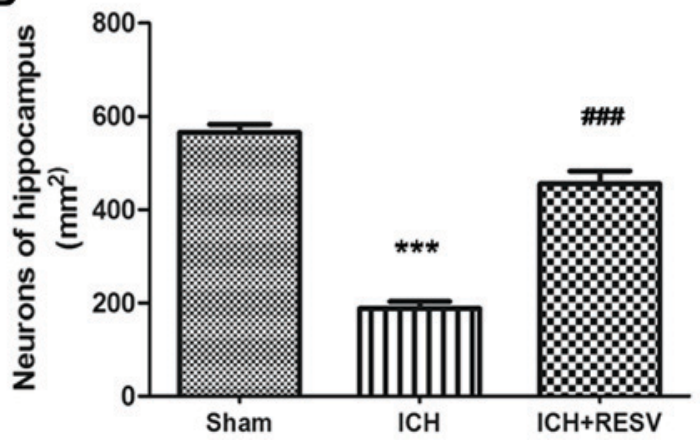

Figure 4. RESV alleviated damage to neurons in the hippocampus region. (A) Neurons in the hippocampus were stained with Nissl 14 days following ICH and microscopic images were captured; magnification, $\mathrm{x} 400$. The image in the black square of lower panel is in the same region as the images in the upper three panels; magnification, x100. The image in the lower panel is an example image of the area. Blue neuronal cells with an articulate structure, exhibiting mottled nuclei and cytoplasm, were detected in the control group (black arrow), while injured neurons with condensed and irregular cytons, alongside surviving neurons were identified in the ICH group (black arrowhead). (B) Quantitative analysis of neuronal cells. The number of neurons was decreased in the ICH group, compared with the sham group. The survival rate of neurons in the ICH+RESV group was greater compared with the ICH group. Scale bar, $50 \mu \mathrm{m}$. Data are presented as the mean \pm standard deviation. ${ }^{* * *} \mathrm{P}<0.001$ vs. sham group, ${ }^{\# \# \#} \mathrm{P}<0.001$ vs. ICH group. RESV, resveratrol; ICH, intracerebral hemorrhage.

A

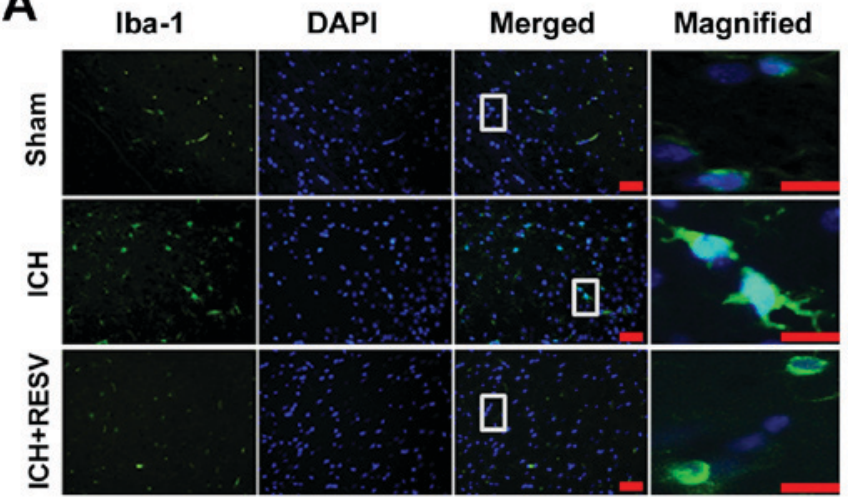

B

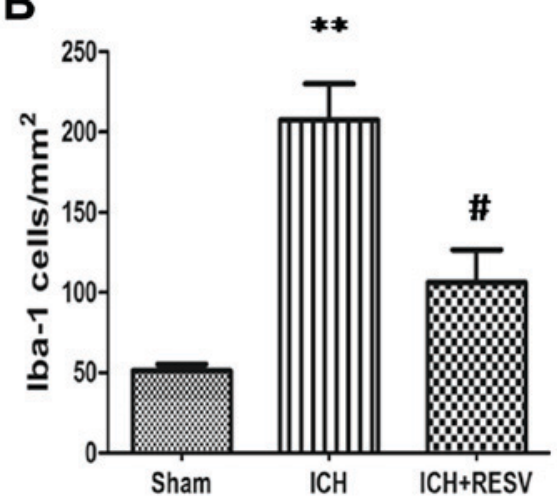

Figure 5. Microglial activation, induced by ICH, was attenuated by RESV. (A) Immunofluorescence staining of Iba-1 in the cortex 14 days post-ICH (magnification, x100). White squares on merged images were magnified (magnification, x400) and presented in the right panel. DNA and Iba-1 were stained blue and green, respectively. (B) Quantification of Iba-1 positive cells. Scale bar, $50 \mu \mathrm{m}$. Data are presented as the mean \pm standard deviation. ${ }^{* *} \mathrm{P}<0.01$ vs. sham group, ${ }^{\#} \mathrm{P}<0.05$ vs. ICH group. ICH, intracerebral hemorrhage; RESV, resveratrol; Iba-1, ionized calcium binding adaptor molecule 1.

by neuroinflammation, the overall size and soma of microglia increase and the thickness of axons increases. Iba-1, a biomarker of microglia, stains microglia green color and revealed that the neurons comprise of a soma and several axons (23). Furthermore, Iba-1 identifies the disperse axons involved in nerve conduction (24). The morphological and proliferative changes of microglia following ICH were determined using Iba-1 to assess whether the neuroprotective effects of RESV were mediated by the anti-inflammatory response of glial cells. Iba-1-stained microglia were activated by the formation of an intracerebral hematoma following ICH (Fig. 5A). The number of microglia and length of microglial axons were significantly increased following ICH $(\mathrm{P}<0.01$; Fig. $5 \mathrm{~B})$. However, the administration of RESV post-surgery significantly reversed the upregulation of ICH induced Iba-1 ( $\mathrm{P}<0.05$; Fig. 5B). These data indicate that the neuroinflammatory response induced by $\mathrm{ICH}$ is ameliorated following RESV treatment.

\section{Discussion}

Previous studies have demonstrated that secondary brain injury, rather than primary mechanical injury, contributes to 
Table I. Comparison of drugs used to treat ICH.

\begin{tabular}{lllll}
\hline Treatment & \multicolumn{1}{c}{ CEGI } & \multicolumn{1}{c}{ GM-1 } & \multicolumn{1}{c}{ BNDF } & \multicolumn{1}{c}{ RESV } \\
\hline Neurological & 4 ml/kg CEGI improves & GM-1 improves & F3. BDNF improves & RESV improves \\
outcome & neurobehavioral outcomes & neurobehavioral outcomes & neurobehavioral & neurobehavioral \\
& 7 days PS (modified & 14 days PS (corner turn & outcomes 8 days & outcomes 7 days PS \\
& Garcia scale) (41) & test) (41) & $\begin{array}{l}\text { PS (mouse ICH model, } \\
\text { (open field)/14 days PS } \\
\text { (Rota-rod) }\end{array}$
\end{tabular}

ICH, intracerebral hemorrhage; CEGI, cattle encephalon glycoside and ignotin; GM-1 monosialotetrahexosyl ganglioside; F3. BNDF, human neural stem cells expressing brain-derived neurotrophic factor; RESV, resveratrol; PS, post-surgery.

the serious complications that occur following ICH $(25,26)$. Excessive bleeding of blood vessels within the brain activates the coagulation cascade leading to the production of thrombin, which may induce the release of pro-inflammatory cytokines, including interleukin-1 $\beta$ (IL-1 $\beta$ ) and tumor necrosis factor $\alpha$ $(\mathrm{TNF}-\alpha)$. This may, in turn, lead to the activation of microglia and other pro-inflammatory molecules (27). The inflammatory response may therefore exacerbate neuronal impairment within the brain. Thus, novel therapeutic strategies to treat the secondary brain injury that occurs following ICH are required to improve the mortality and disability rates of patients with ICH (28).

It has been demonstrated that RESV may attenuate neurological deficits in certain diseases that results in brain injury (29-32). However, to the best of our knowledge, no studies have assessed the effects of RESV on brain injury induced by ICH. Thus, the present study examined the neuroprotective function of RESV on ICH in rats.

The rotarod test used in the present study indicated that $100 \mathrm{mg} / \mathrm{kg}$ RESV administered intraperitoneally for 2 weeks stimulated the recovery of motor abilities following ICH. The recovery time with RESV administration was decreased when compared with a previous study (33). This unexpected result may have been due to higher doses of RESV being used in the present study. In the open field test, ICH rats treated with RESV were more active than those in the ICH group. Furthermore, rats in the ICH+RESV group travelled a greater distance and at a higher speed than rats in the ICH group 7 days post-surgery. This indicates that RESV treatment exhibits a positive effect on the recovery of rat motor abilities following ICH. However, a decrease in the total distance traveled and average speed was observed 14 days post-surgery among all groups, which may have been due to the habituation of rats to the experimental environment over repeated exposure to the open field test, which was also documented in a previous study (34). Thus, future studies may require more behavioral tests, including the water maze, balance beam and contralateral hindlimb retraction tests to assess motor functions, to limit the likelihood of habituation.

In addition, the present study demonstrated that the number of Nissl-stained neurons in the hippocampus was significantly increased following 2 weeks RESV treatment. This was consistent with the results of previous studies, which demonstrated that RESV reduces cell loss, inhibits blood brain barrier disruption and decreases edema following brain injury $(29,35)$. The behavioral performances of rats were also improved following an increase in neural cell survival rate. This may help to explain the results of the present study. In addition, the number of activated microglia decreased following RESV treatment post-ICH, which was in accordance with the anti-inflammatory function of RESV identified in previous studies $(36,37)$. The RESV-induced downregulation of brain immune cell activation via the inhibition of transcriptional factors, including peroxisome proliferator-activated receptor $\alpha$ (38) and nuclear factor- $\kappa \mathrm{B}$ have also been identified (39). However, it remains unclear whether the expression of certain downstream inflammatory factors, including matrix metalloproteinase 9, IL- $1 \beta$ and TNF- $\alpha$, are regulated by RESV. The expression of astrocytes should also be examined in order to compare the function of these immune cells during neuroinflammation. Further studies are therefore required to elucidate the exact molecular mechanism of RESV.

Clinical trials have demonstrated that RESV is safe to use (40), however, side effects associated with high doses of RESV remains a challenge. For this reason, RESV has not yet been approved by the food and drug association for use in the treatment of ICH. Therefore, the toxicological properties of RESV should be investigated in future studies. However, clinical trials have demonstrated that RESV has a greater effect than cattle encephalon glycoside and ignotin (41), monosialotetrahexosyl ganglioside (41) and human neural stem cells expressing brain-derived neurotrophic factor (42) in the treatment of ICH (Table I). Thus, RSEV may be an appropriate candidate to treat patients with ICH.

In conclusion, the present study demonstrated that RESV improves rat motor abilities and deactivates the neuroinflammatory response following ICH. These results indicate that treatment with $100 \mathrm{mg} / \mathrm{kg}$ RESV attenuates the neurological deficit caused by ICH and may be used as a novel therapeutic agent to treat $\mathrm{ICH}$.

\section{Acknowledgements}

The authors would like to thank Dr Feng Yin (Key Laboratory of Chemical Genomics, School of Chemical Biology and Biotechnology, Peking University Shenzhen Graduate School) for the critical reading of the manuscript and Dr Jun Ju (Key Laboratory of Chemical Genomics, School of Chemical Biology and Biotechnology, Peking University Shenzhen Graduate School) for the assistance in image processing. 


\section{Funding}

The present study was supported by the Shandong Provincial Medical and Health Development Projects of China (grant no. 2014WS0012) and the Natural Science Foundation of Guangdong Province (grant. no. 2014A030313779). The funders had no role in study design, data collection and interpretation, or the decision to submit the work for publication.

\section{Availability of data and materials}

The datasets generated and/or analyzed during the current study are not publicly available due to the regulations of the researched funding but are available from the corresponding author on reasonable request.

\section{Authors' contributions}

JCC, WL and FL proposed the hypothesis, analyzed the results, and wrote the manuscript. JCC designed and executed the majority of the experiments. WBK, XXZ, PM, CXL and YW assisted in the execution of some experiments. All authors read and approved the final manuscript.

\section{Ethics approval and consent to participate}

All experimental procedures were approved by the Animal Care and Use Committee of Peking University Shenzhen Graduate School (Shenzhen, China).

\section{Consent for publication}

Not applicable.

\section{Competing interests}

The authors declare that they have no competing interests.

\section{References}

1. Rojas H, Lekic T, Chen W, Jadhav V, Titova E, Martin RD, Tang $\mathrm{J}$ and Zhang $\mathrm{J}$ : The antioxidant effects of melatonin after intracerebral hemorrhage in rats. Acta Neurochir Suppl 105: 19-21, 2008.

2. Wu CH, Yang RL, Huang SY, Li HZ, Wang KY, Yang DH, Yan XH, Xue XH, Wu SY, Wang JM, et al: Analysis of thrombin-antithrombin complex contents in plasma and hematoma fluid of hypertensive intracerebral hemorrhage patients after clot removal. Eur J Neurol 18: 1060-1066, 2011.

3. Kitaoka T, Hua Y, Xi G, Hoff JT and Keep RF: Delayed argatroban treatment reduces edema in a rat model of intracerebral hemorrhage. Stroke 33: 3012-3018, 2002.

4. Gu Y, Gong Y, Liu WQ, Keep RF, Xi G and Hua Y: Zinc protoporphyrin attenuates white matter injury after intracerebral hemorrhage. Acta Neurochir Suppl 121: 199-202, 2016.

5. Fung C, Murek M, Klinger-Gratz PP, Fiechter M, Z'Graggen WJ, Gautschi OP, El-Koussy M, Gralla J, Schaller K, Zbinden M, et al: Effect of decompressive craniectomy on perihematomal edema in patients with intracerebral hemorrhage. PLoS One 11: e0149169, 2016.

6. Dragone T, Cianciulli A, Calvello R, Porro C, Trotta T and Panaro MA: Resveratrol counteracts lipopolysaccharide-mediated microglial inflammation by modulating a SOCS-1 dependent signaling pathway. Toxicol In Vitro 28: 1126-1135, 2014.
7. Bobermin LD, Wartchow KM, Flores MP, Leite MC, Quincozes-Santos A and Goncalves CA: Ammonia-induced oxidative damage in neurons is prevented by resveratrol and lipoic acid with participation of heme oxygenase 1. Neurotoxicology 49: 28-35, 2015.

8. Huang T, Gao D, Jiang X, Hu S, Zhang L and Fei Z: Resveratrol inhibits oxygen-glucose deprivation-induced MMP-3 expression and cell apoptosis in primary cortical cells via the NF- $\kappa \mathrm{B}$ pathway. Mol Med Rep 10: 1065-1071, 2014.

9. Nguyen JK, Jorfi M, Buchanan KL, Park DJ, Foster EJ, Tyler DJ, Rowan SJ, Weder C and Capadona JR: Influence of resveratrol release on the tissue response to mechanically adaptive cortical implants. Acta Biomater 29: 81-93, 2016.

10. Virgili M and Contestabile A: Partial neuroprotection of in vivo excitotoxic brain damage by chronic administration of the red wine antioxidant agent, trans-resveratrol in rats. Neurosci Lett 281: 123-126, 2000.

11. Ren J, Fan C, Chen N, Huang J and Yang Q: Resveratrol pretreatment attenuates cerebral ischemic injury by upregulating expression of transcription factor $\mathrm{Nrf} 2$ and $\mathrm{HO}-1$ in rats. Neurochem Res 36: 2352-2362, 2011.

12. Liu C, Shi Z, Fan L, Zhang C, Wang K and Wang B: Resveratrol improves neuron protection and functional recovery in rat model of spinal cord injury. Brain Res 1374: 100-109, 2011.

13. Davinelli S, Sapere N, Zella D, Bracale R, Intrieri M and Scapagnini G: Pleiotropic protective effects of phytochemicals in Alzheimer's disease. Oxid Med Cell Longev 2012: 386527, 2012.

14. Yu Y, Wang R, Chen C, Du X, Ruan L, Sun J, Li J, Zhang L, O'Donnell JM, Pan J and Xu Y: Antidepressant-like effect of trans-resveratrol in chronic stress model: Behavioral and neurochemical evidences. J Psychiatr Res 47: 315-322, 2013.

15. Della-Morte D, Dave KR, DeFazio RA, Bao YC, Raval AP and Perez-Pinzon MA: Resveratrol pretreatment protects rat brain from cerebral ischemic damage via a sirtuin 1-uncoupling protein 2 pathway. Neuroscience 159: 993-1002, 2009.

16. Price NL, Gomes AP, Ling AJ, Duarte FV, Martin-Montalvo A, North BJ, Agarwal B, Ye L, Ramadori G, Teodoro JS, et al: SIRT1 is required for AMPK activation and the beneficial effects of resveratrol on mitochondrial function. Cell Metab 15: 675-690, 2012.

17. Ungvari Z, BagiZ, Feher A, Recchia FA, Sonntag WE, Pearson K, de Cabo R and Csiszar A: Resveratrol confers endothelial protection via activation of the antioxidant transcription factor Nrf2. Am J Physiol Heart Circ Physiol 299: H18-H24, 2010.

18. Deinsberger W, Vogel J, Kuschinsky W, Auer LM and Böker DK: Experimental intracerebral hemorrhage: Description of a double injection model in rats. Neurol Res 18: 475-477, 1996.

19. National Research Council: Guide for the Care and Use of Laboratory Animals. 8th edition. National Acadamies Press, Washington, DC, 2011.

20. Shao A, Wu H, Hong Y, Tu S, Sun X, Wu Q, Zhao Q, Zhang J and Sheng J: Hydrogen-rich saline attenuated subarachnoid hemorrhage-induced early brain injury in rats by suppressing inflammatory response: Possible involvement of NF- $\kappa \mathrm{B}$ pathway and NLRP3 inflammasome. Mol Neurobiol 53: 3462-3476, 2016.

21. Lee HI, Park JH, Park MY, Kim NG, Park KJ, Choi BT, Shin YI and Shin HK: Pre-conditioning with transcranial low-level light therapy reduces neuroinflammation and protects blood-brain barrier after focal cerebral ischemia in mice. Restor Neurol Neurosci 34: 201-214, 2016.

22. Kettenmann H, Hanisch UK, Noda M and Verkhratsky A: Physiology of microglia. Physiol Rev 91: 461-553, 2011.

23. Tischer J, Krueger M, Mueller W, Staszewski O, Prinz M, Streit WJ and Bechmann I: Inhomogeneous distribution of Iba-1 characterizes microglial pathology in Alzheimer's disease. Glia 64: 1562-1572, 2016.

24. Ahmed Z, Shaw G, Sharma VP, Yang C, McGowan E and Dickson DW: Actin-binding proteins coronin-1a and IBA-1 are effective microglial markers for immunohistochemistry. J Histochem Cytochem 55: 687-700, 2007.

25. Ducruet AF, Zacharia BE, Hickman ZL, Grobelny BT, Yeh ML, Sosunov SA and Connolly ES Jr: The complement cascade as a therapeutic target in intracerebral hemorrhage. Exp Neurol 219: 398-403, 2009.

26. Thiex R and Tsirka SE: Brain edema after intracerebral hemorrhage: Mechanisms, treatment options, management strategies, and operative indications. Neurosurg Focus 22: E6, 2007. 
27. Hua Y, Wu J, Keep RF, Nakamura T, Hoff JT and Xi G: Tumor necrosis factor-alpha increases in the brain after intracerebral hemorrhage and thrombin stimulation. Neurosurgery 58: 542-550, 2006

28. Xi G, Keep RF and Hoff JT: Mechanisms of brain injury after intracerebral haemorrhage. Lancet Neurol 5: 53-63, 2006.

29. Shao AW, Wu HJ, Chen S, Ammar AB, Zhang JM and Hong Y: Resveratrol attenuates early brain injury after subarachnoid hemorrhage through inhibition of NF- $\mathrm{kB}$-dependent inflammatory/MMP-9 pathway. CNS Neurosci Ther 20: 182-185, 2014

30. Koronowski KB, Dave KR, Saul I, Camarena V, Thompson JW, Neumann JT, Young JI and Perez-Pinzon MA: Resveratrol preconditioning induces a novel extended window of ischemic tolerance in the mouse brain. Stroke 46: 2293-2298, 2015.

31. Lin CJ, Chen TH, Yang LY and Shih CM: Resveratrol protects astrocytes against traumatic brain injury through inhibiting apoptotic and autophagic cell death. Cell Death Dis 5: e1147, 2014.

32. Ghaiad HR, Nooh MM, El-Sawalhi MM and Shaheen AA: Resveratrol promotes remyelination in cuprizone model of multiple sclerosis: Biochemical and histological study. Mol Neurobiol 54: 3219-3229, 2017.

33. Liu L, Wang S, Xu R, Zheng J, Tang J, Tang X and Zhang D: Experimental intracerebral haemorrhage: Description of a semi-coagulated autologous blood model in rats. Neurol Res 37: 874-879, 2015.

34. Paylor R, Spencer CM, Yuva-Paylor LA and Pieke-Dahl S: The use of behavioral test batteries, II: Effect of test interval. Physiol Behav 87: 95-102, 2006.

35. Arteaga O, Revuelta M, Urigüen L, Álvarez A, Montalvo H and Hilario E: Pretreatment with resveratrol prevents neuronal injury and cognitive deficits induced by perinatal hypoxia-ischemia in rats. PLoS One 10: e0142424, 2015.
36. Zhang X, Wu Q, Zhang Q, Lu Y, Liu J, Li W, Lv S, Zhou M, Zhang $X$ and Hang C: Resveratrol attenuates early brain injury after experimental subarachnoid hemorrhage via inhibition of NLRP3 inflammasome activation. Front Neurosci 11: 611, 2017.

37. Zhang XS, Li W, Wu Q, Wu LY, Ye ZN, Liu JP, Zhuang Z, Zhou ML, Zhang X and Hang CH: Resveratrol attenuates acute inflammatory injury in experimental subarachnoid hemorrhage in rats via inhibition of TLR4 pathway. Int J Mol Sci 17: pii:E1331, 2016.

38. Ayub A, Poulose N and Raju R: Resveratrol improves survival and prolongs life following hemorrhagic shock. Mol Med 21: 305-312, 2015.

39. Zhang Q, Yuan L, Zhang Q, Gao Y, Liu G, Xiu M, Wei X, Wang Z and Liu D: Resveratrol attenuates hypoxia-induced neurotoxicity through inhibiting microglial activation. Int Immunopharmacol 28: 578-587, 2015.

40. Turner RS, Thomas RG, Craft S, van Dyck CH, Mintzer J, Reynolds BA, Brewer JB, Rissman RA, Raman R, Aisen PS, et al: A randomized, double-blind, placebo-controlled trial of resveratrol for Alzheimer disease. Neurology 85: 1383-1391, 2015.

41. Li R, Ma K, Zhao H, Feng Z, Yang Y, Ge H, Zhang X, Tang J, Yin Y, Liu X, et al: Cattle encephalon glycoside and ignotin reduced white matter injury and prevented post-hemorrhagic hydrocephalus in a rat model of intracerebral hemorrhage. Sci Rep 6: 35923, 2016.

42. Lee HJ, Lim IJ, Lee MC and Kim SU: Human neural stem cells genetically modified to overexpress brain-derived neurotrophic factor promote functional recovery and neuroprotection in a mouse stroke model. J Neurosci Res 88: 3282-3294, 2010.

c) (i) $\Theta$ This work is licensed under a Creative Commons Attribution-NonCommercial-NoDerivatives 4.0 International (CC BY-NC-ND 4.0) License. 